\title{
ANGUSTIFOLIA is a central component of tissue morphogenesis mediated by the atypical receptor-like kinase STRUBBELIG
}

Yang Bai ${ }^{1,3+}$, Prasad Vaddepalli ${ }^{2 \dagger}$, Lynette Fulton ${ }^{2,4 \dagger}$, Hemal Bhasin $^{1 \dagger}$, Martin Hülskamp $^{1}$ and Kay Schneitz ${ }^{2 *}$

\begin{abstract}
Background: During plant tissue morphogenesis cells have to coordinate their behavior to allow the generation of the size, shape and cellular patterns that distinguish an organ. Despite impressive progress the underlying signaling pathways remain largely unexplored. In Arabidopsis thaliana, the atypical leucine-rich repeat receptor-like kinase STRUBBELIG (SUB) is involved in signal transduction in several developmental processes including the formation of carpels, petals, ovules and root hair patterning. The three STRUBBELIG-LIKE MUTANT (SLM) genes DETORQUEO (DOQ), QUIRKY (QKY) and ZERZAUST (ZET) are considered central elements of SUB-mediated signal transduction pathways as corresponding mutants share most phenotypic aspects with sub mutants.

Results: Here we show that DOQ corresponds to the previously identified ANGUSTIFOLIA gene. The genetic analysis revealed that the doq-1 mutant exhibits all additional mutant phenotypes and conversely that other an alleles show the $s / m$ phenotypes. We further provide evidence that SUB and AN physically interact and that AN is not required for subcellular localization of SUB.

Conclusions: Our data suggest that AN is involved in SUB signal transduction pathways. In addition, they reveal previously unreported functions of $A N$ in several biological processes, such as ovule development, cell morphogenesis in floral meristems, and root hair patterning. Finally, SUB and AN may directly interact at the plasma membrane to mediate SUB-dependent signaling.
\end{abstract}

Keywords: ANGUSTIFOLIA, Flower, Ovule, Receptor-like kinase, Root hair patterning, Signal transduction, Tissue morphogenesis, STRUBBELIG

\section{Background}

Tissue morphogenesis and cellular patterning require extensive cellular communication. In plants the coordination of cellular behavior within a tissue is intrinsically linked to cell wall biogenesis and dynamics, as plant cells are connected through semi-rigid cell walls that drastically limit their relative movement. It is a major current challenge in plant biology to understand the mechanistic basis of intercellular communication and its connection to the cell wall during tissue morphogenesis.

\footnotetext{
*Correspondence: schneitz@wzw.tum.de

${ }^{\dagger}$ Equal contributors

2Entwicklungsbiologie der Pflanzen, Wissenschaftszentrum Weihenstephan, Technische Universität München, Emil-Ramann-Str. 4, Freising 85354, Germany

Full list of author information is available at the end of the article
}

In Arabidopsis, intercellular signaling mediated by the atypical leucine-rich repeat transmembrane receptor-like (LRR-RLK) STRUBBELIG (SUB) is essential for a number of developmental processes [1-6]. Ovules of sub mutants show frequent defects in the initiation and outgrowth of the outer integument. In addition, sub mutants exhibit twisted stems, petals and carpels/siliques. These phenotypes indicate a role for $S U B$ in the control of integument initiation and outgrowth as well as stem and floral organ shape [1,2,6]. SUB also plays a role in internode length (and thus stem height), a trait that is potentially important for optimizing yield in crop plants.

At the cellular level, frequent misorientations of cell division planes were observed in e.g. L1 and L2 cells of young apical and floral meristems of sub mutants. Therefore, it was postulated that SUB signaling plays a role in orienting cell division planes in initiating 
integuments and floral meristems and thus influences the morphogenetic behavior of cells in a tissue context [1]. In addition, $S U B$, also known as SCRAMBLED $(S C M)$, is involved in root hair patterning [3,4]. In this context, sub mutations lead to a randomization of root hair patterning such that root hairs develop ectopically or are not formed in the correct files.

In accordance with a perceived role of $S U B$ in coordinating cellular behavior in tissue morphogenesis and cell patterning, $S U B$ acts in a non-cell-autonomous fashion and mediates inter-cell-layer signaling across histogenic cell layers in the ovule, the floral meristem [5] and the $\operatorname{root}[7]$.

SUB belongs to the LRRV/STRUBBELIG-RECEPTOR FAMILY (SRF) family $[8,9]$ and has several protein domains including an extracellular domain with seven leucine-rich repeats, a transmembrane domain and a cytoplasmic putative kinase domain $[1,3,6]$. Interestingly, a set of biochemical and genetic data indicated that although the kinase domain is essential for SUB function, enzymatic phosphotransfer activity is not $[1,6]$. Thus, SUB is likely a so-called atypical or dead kinase.

Signaling by atypical kinases is poorly understood in plants $[10,11]$. In addition, a detailed structure-function analysis of $S U B$ suggested that the organ or cell-specific aspects of SUB-mediated signaling are not integrated at the SUB receptor, but involve other components that act together with, or downstream of SUB [6]. In order to unravel the signal-transduction pathway of SUB we have previously identified three complementation groups sharing the $s u b$-like mutant $(s l m)$ phenotypes [2]. In addition, it was found that there is significant overlap in $S L M$-sensitive gene expression. Taken together the results indicated that $S L M$ genes contribute to $S U B$ signal transduction. The corresponding genes are called QUIRKY (QKY), ZERZAUST (ZET), and DETORQUEO (DOQ) [2]. Initial molecular analysis suggested that $Q K Y$ encodes a putative membranelocalized protein with four $\mathrm{C} 2$ domains thus potentially connecting SUB to membrane-associated $\mathrm{Ca}^{2+}$ - and phospholipid-dependent signaling [2].

In this work we focused on the $D O Q$ gene. We show that doq-1 is a mutant allele of the ANGUSTIFOLIA $(A N)$ gene. The doq-1 mutant carries a point mutation in the $A N$ gene and we further demonstrate that doq-1 shares phenotypes with other an alleles and conversely, other an alleles show all slm phenotypes tested. These results rule out the possibility that $d o q-1$ is an atypical allele. In addition, we provide evidence that SUB and AN can physically interact and that $A N$ does not influence subcellular SUB distribution. Together our results reveal that $A N$ is involved in $S U B$-dependent signaling events.

\section{Results}

doq-1 mutants exhibit an underbranched trichome and narrow leaf phenotype

Meiotic recombination mapping placed $D O Q$ in a $330.6 \mathrm{~kb}$ interval at the top of chromosome 1 (see Methods). This interval included $A N$, a gene previously described to affect trichome branching, leaf morphology, and silique shape [12-15]. During the course of this analysis we noticed that doq-1 trichomes are underbranched. Together with the previously described narrow leaf phenotype of doq-1 [2], this suggested that $D O Q$ and $A N$ functions are related. We therefore compared the doq-1 mutant leaf and trichome phenotype with three reference $a n$ alleles, $a n-1, a n-2$, an-EM1 and two an-2 35S::YFP:AN rescued lines. In doq-1 mutants, reductions in trichome branching approached levels seen in the an-1, an-2, an-EM1 alleles (Figure 1, Table 1). Two-branched and three-branched trichomes are almost absent and a new class of unbranched trichomes was observed. The leaf shape of doq-1 mutants was also similar to that seen in an-1, an-2, an-EM1 mutants (Figure 2). Correspondingly and in step with the reference an alleles, the leaf length/width ratio in doq-1 was significantly increased (Table 2).

\section{The $D O Q$ gene corresponds to the $A N$ gene}

We tested whether the $D O Q$ gene corresponds to the $A N$ gene. Genetic analysis revealed no complementation of $d o q-1$ with an-1 indicating that $d o q-1$ is allelic to $a n$. Furthermore, we sequenced the $A N$ gene in the doq-1 mutant and demonstrated a $\mathrm{G}$ to $\mathrm{A}$ transition at position 509 in the cDNA coding region. This mutation causes a glycine to aspartic acid substitution at position 170 that is located in the predicted $\mathrm{NAD}(\mathrm{P})$-binding domain.

\section{an alleles show s/m phenotypes}

As most $\operatorname{slm}$ phenotypes had not been reported for an mutants, the question arose whether $d o q-1$ is an atypical an allele showing new phenotypes or whether all an alleles share the $\operatorname{slm}$ phenotypes. We therefore compared $s l m$ phenotypes between $d o q-1$, the $a n-1, a n-2, a n-E M 1$ alleles and $a n-2$ 35S::YFP:AN rescued lines.

As described for $d o q-1$, all $a n$ alleles showed premature opening of flowers and twisted petals (Figure 3). These phenotypes are rescued in the an-2 35S::YFP:AN lines. In addition, doq-1-like twisting of siliques was observed in all an alleles and could be rescued by $A N$ overexpression (Figure 4). Plants carrying the doq-1 mutation show a weak ovule phenotype as compared to $s u b$ mutants [2]. To study the cellular patterns in an ovules we used Scanning Electron Microscopy. We observed smaller cells with atypical division planes at the distal end of the outer integument in all $a n$ alleles tested and a rescue of this phenotype in an-2 35S::YFP:AN lines (Figure 5). Finally, we analyzed the role of $A N$ in root hair patterning. 

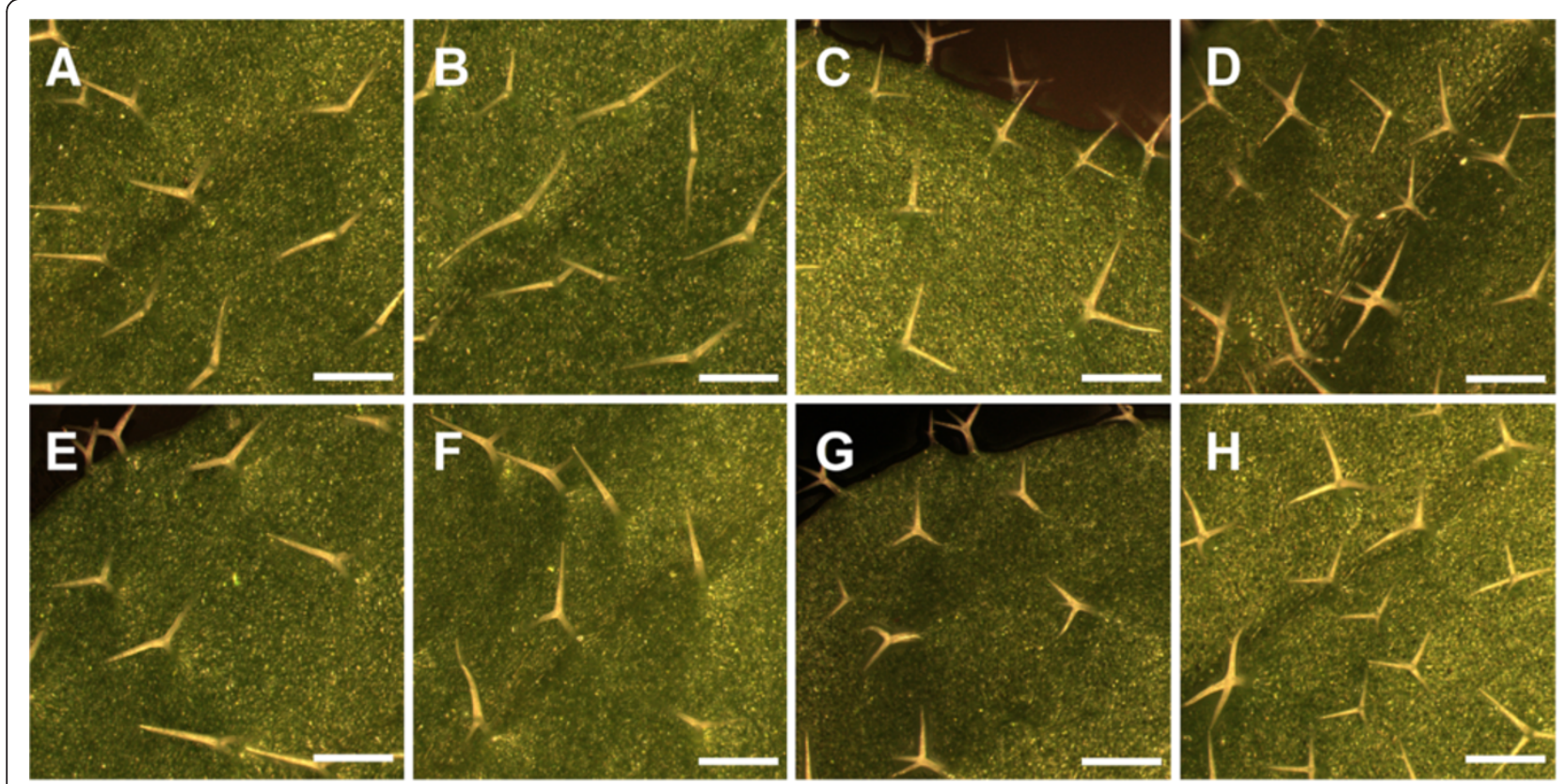

Figure 1 Trichome branching in WT, an mutants and rescued lines. Trichomes in the an-1 mutant (A), the an-2 mutant (B), the an-2 35S::YFP: AN \#2 line (C), the an-2 35S::YFP:AN \#4 line (D), the doq-1 mutant (E), the an-EM1 mutant (F), WT Ler $(\mathbf{G})$ and WT Col-0 (H). Scale bars: $400 \mu \mathrm{m}$.

To this end we investigated the expression profiles of a GL2:GUS construct in an-1, an-2, an-EM1 allelic backgrounds. GL2:GUS is expressed in atrichoblast cell files and serves as a convenient marker for root hair patterning [16]. Mutant an-1, an-2, an-EM1 alleles displayed a severe distortion of the GL2:GUS pattern (Figure 6). Thus $A N$ is a new component of the root hair patterning machinery.

\section{SUB and AN can interact directly}

Next we addressed in more detail how $S U B$ and DOQ/ $A N$ relate to each other during $S U B$-dependent signal transduction. Earlier results indicated that the two genes do not regulate each other at the transcriptional level [2]. We thus tested if DOQ/AN and SUB have the potential to interact directly at the protein level. Indeed, the intra-cellular domain of SUB (SUBICD, residues 371 to 768), but not the extra-cellular domain (SUBECD, residues 26 to 340), was able to interact with $\mathrm{AN}$ in a yeast two-hybrid assay (Figure 7). In addition, fusions of maltosebinding protein (MBP) to SUBICD and the SUB fulllength proteins were able to interact with a fusion of glutathione-S-transferase (GST) to AN in in vitro pulldown assays involving bacterially-expressed recombinant proteins (Figure 7).

\section{$A N$ is not required for subcellular localization of SUB:EGFP} in roots

Previous studies involving a functional SUB:EGFP fusion protein indicated that SUB is localized to the plasma membrane and undergoes brefeldin A (BFA)-sensitive recycling [5-7]. As the AN homolog in animals was identified as a Brefeldin A ribosylated substrate [17] we tested whether the subcellular localization of SUB is dependent on $D O Q / A N$ function. Towards this end we analyzed expression of a functional SUB::SUB:EGFP reporter [6] in roots of $d o q-1$ mutants in the absence or presence of BFA (Figure 8). Expression of the reporter appeared normal under both experimental conditions indicating that $A N$ is not required for correct subcellular localization and recycling of SUB.

Table 1 Frequency of trichomes with different branch numbers in WT and an mutant lines on the fifth rosette leaves

\begin{tabular}{lllll}
\hline Genotype $^{\text {a }}$ & unbranched & $\begin{array}{l}\text { One } \\
\text { branch }\end{array}$ & $\begin{array}{l}\text { Two } \\
\text { branches }\end{array}$ & $\begin{array}{l}\text { Three } \\
\text { branches }\end{array}$ \\
\hline Ler & 0 & 2.8 & 95.2 & 2.0 \\
Col-0 & 0 & 1.7 & 83.3 & 15.0 \\
an-EM1 & 1.9 & 96.2 & 1.9 & 0 \\
doq-1 & 2.0 & 97.0 & 1.0 & 0 \\
an-1 & 4.8 & 95.2 & 0 & 0 \\
an-2 & 3.6 & 95.4 & 1.0 & 0 \\
an-2 355::YYFP: & 0 & 3.5 & 87.7 & 8.8 \\
AN \#2 & & & & \\
an-2 355::YYFP: & 0 & 2.4 & 88.0 & 9.6 \\
AN\#4 & & & & \\
\hline
\end{tabular}

${ }^{a} n=300$ trichomes for each genotype. 


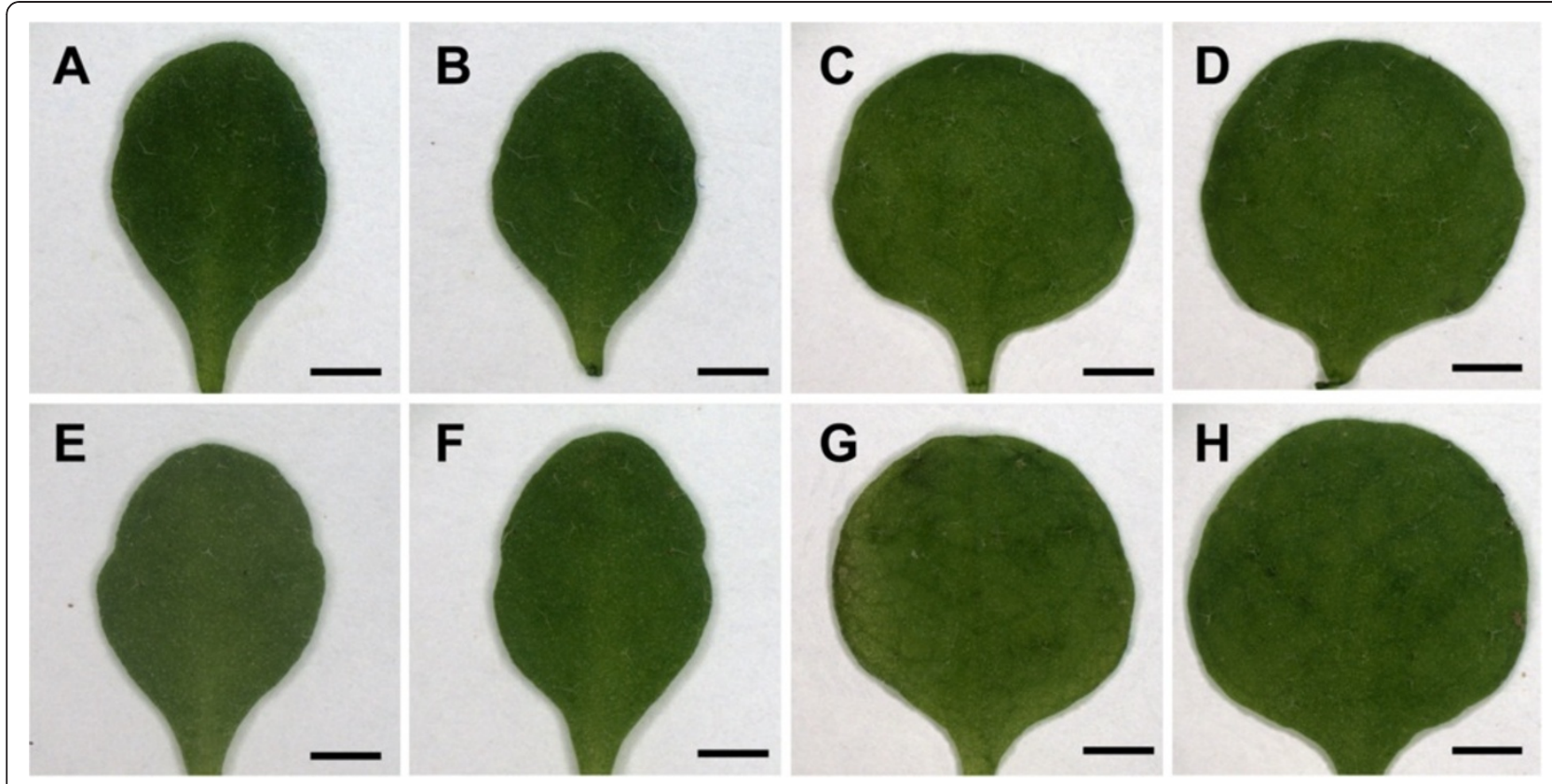

Figure 2 Leaf shape in WT, an mutants and rescued lines. The first pair of rosette leaves in the an-1 mutant (A), the an-2 mutant (B), the an-2 355::YFP:AN \#2 line (C), the an-2 355:::YFP:AN \#4 line (D), the doq-1 mutant $(\mathbf{E})$, the an-EM1 mutant $(\mathbf{F})$, WT Ler $(\mathbf{G})$ and WT Col-0 (H). Scale bars: $1.5 \mathrm{~mm}$.

\section{ERECTA influences the an phenotype}

The sub phenotype is sensitive to the genetic background [1] and it was shown that mutations in ERECTA $(E R)$, encoding a LRR-RLK [18], and SUB interact synergistically as sub er double mutants exhibit strongly reduced plant height [6]. To investigate this issue we transformed pKUT196, a plasmid containing $9.3 \mathrm{~kb}$ of Col-0 genomic DNA spanning the entire ER locus $[18,19]$, into doq-1 (Ler) plants and asked whether a wild-type copy of $E R$ would affect the doq-1 phenotype. Interestingly, the transgene had a similar effect on plant height but not on other aspects of the doq-1 and sub-1 phenotypes (Figure 9) [6]. Similar to $s u b-1$ ER plants, plant height was rescued in doq-1 ER plants indicating that height reduction in $d o q-1$ is caused by a synergistic interaction between the $d o q-1$ and er mutations. It should be noted, however, that plant height in an-2 (Col) was slightly reduced as well, despite the presence of wild-type $E R$, though not to the extent as in doq-1 (Ler). This suggests that $A N$ affects plant height in part independently of $E R$. Full rescue of plant height in doq-1 $E R$ plants indicates the presence of additional modifiers that influence this trait in Ler.

\section{Discussion}

Multiple lines of evidence indicate that $A N$ is involved in the SUB-dependent signaling mechanism. First, our genetic data show that all tested phenotypic aspects of sub are shared by an mutants. Second, DOQ/AN and
$S U B$ influence the expression of a common set of target genes. For example, 62 percent of genes misexpressed in sub flowers are also misexpressed in doq-1/an [2]. Thirdly, AN and SUB are able to interact physically in two different assays. Finally, an and er mutations synergistically affect internode length and plant height, as was observed for sub and er [6]. These results unexpectedly bring together two well-established but previously unconnected research fields. What do we learn for the function of $A N$ and what for the function of SUB?

\section{Table 2 Length and width measurements in rosette} leaves

\begin{tabular}{ll}
\hline Genotype & Ratio $(\mathbf{L} / \mathbf{W})^{\mathbf{a}, \mathbf{b}, \mathbf{c}}$ \\
\hline Ler & $1.08( \pm 0.06)$ \\
Col-0 & $1.03( \pm 0.05)$ \\
an-EM1 & $1.46( \pm 0.07)$ \\
doq-1 & $1.38( \pm 0.08)$ \\
an-1 & $1.42( \pm 0.10)$ \\
an-2 & $1.47( \pm 0.08)$ \\
an-2 35S::YFP:AN \#2 & $1.03( \pm 0.07)$ \\
an-2 35S::YFP:AN\#4 & $1.07( \pm 0.04)$ \\
\hline
\end{tabular}

${ }^{\mathrm{a}}$ The value was calculated from 20 rosette leaves in each lines.

${ }^{\mathrm{b}}$ The ratios are calculated by the formula: ratio=length $(\mathrm{L}) /$ width $(\mathrm{W})$. Values represent mean $\pm \mathrm{SD}$.

'Two-way ANOVA followed by HSD Tukey test. Comparisons were done between individual mutant/wild-type pairs $(p<0.001)$ and between individual an-2 35S::YFP:AN/wild-type pairs ( $p>0.05)$. 

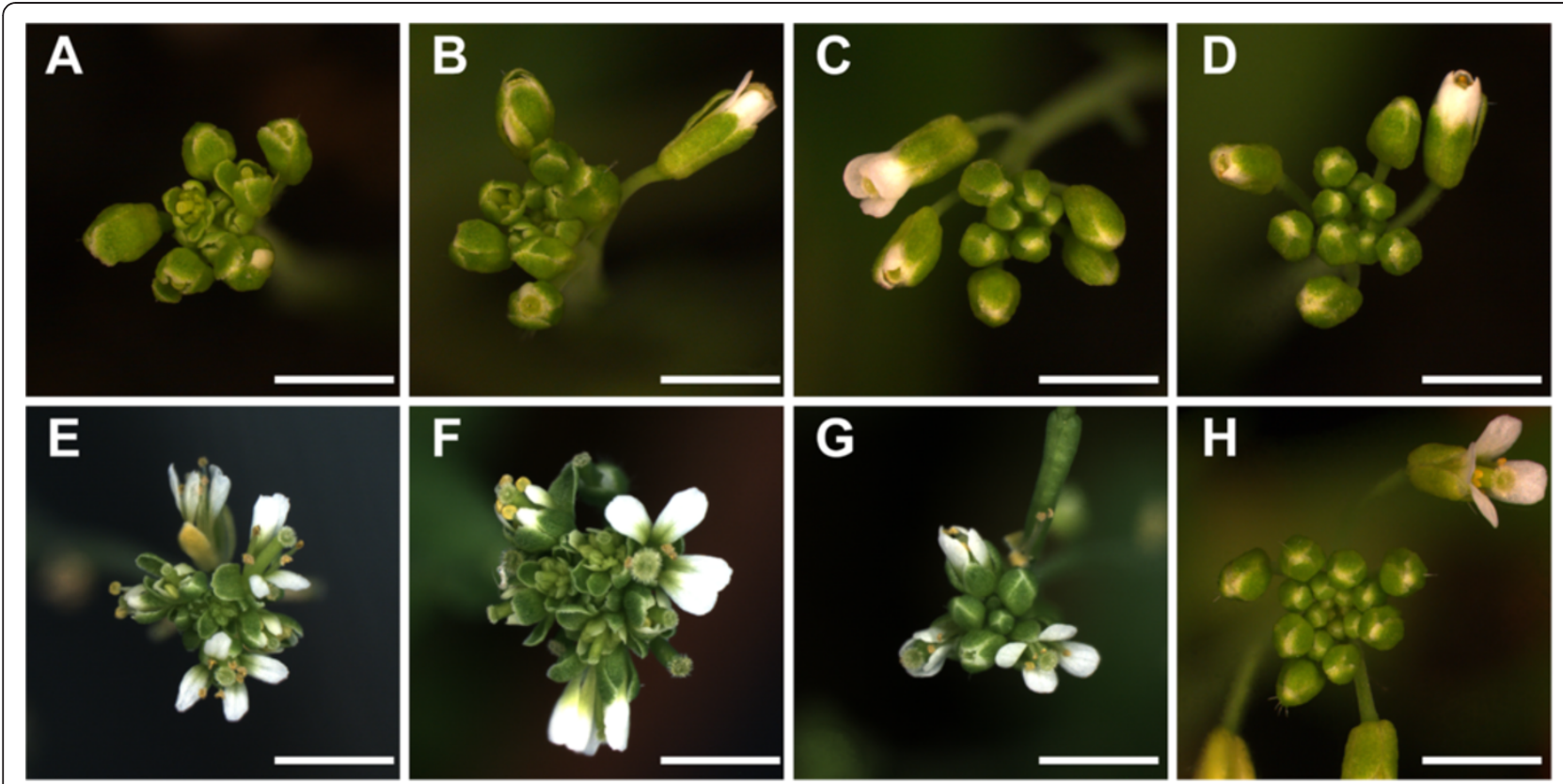

Figure 3 Inflorescence in WT, an mutants and rescued lines. Prematurely opened flower buds in the an-1 mutant (A), the an-2 mutant (B), the doq-1 mutant $(\mathbf{E})$ and the an-EM1 mutant $(\mathbf{F})$. Premature opening of flowers is rescued in the an-2 355::YFF:AN \#2 line (C) and an-2 35S:: YFP:AN \#4 line (D), as compared to WT Ler (G) and WT Col-0 (H). Scale bars: 3 mm.
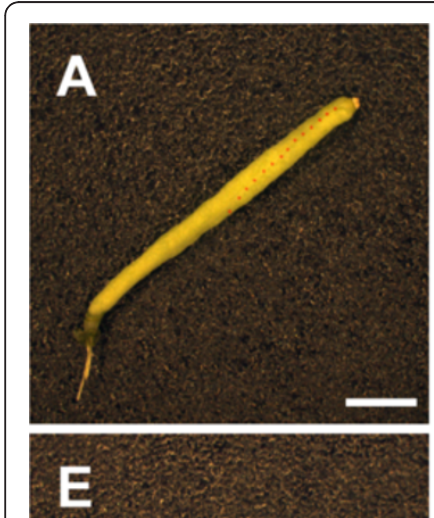

E
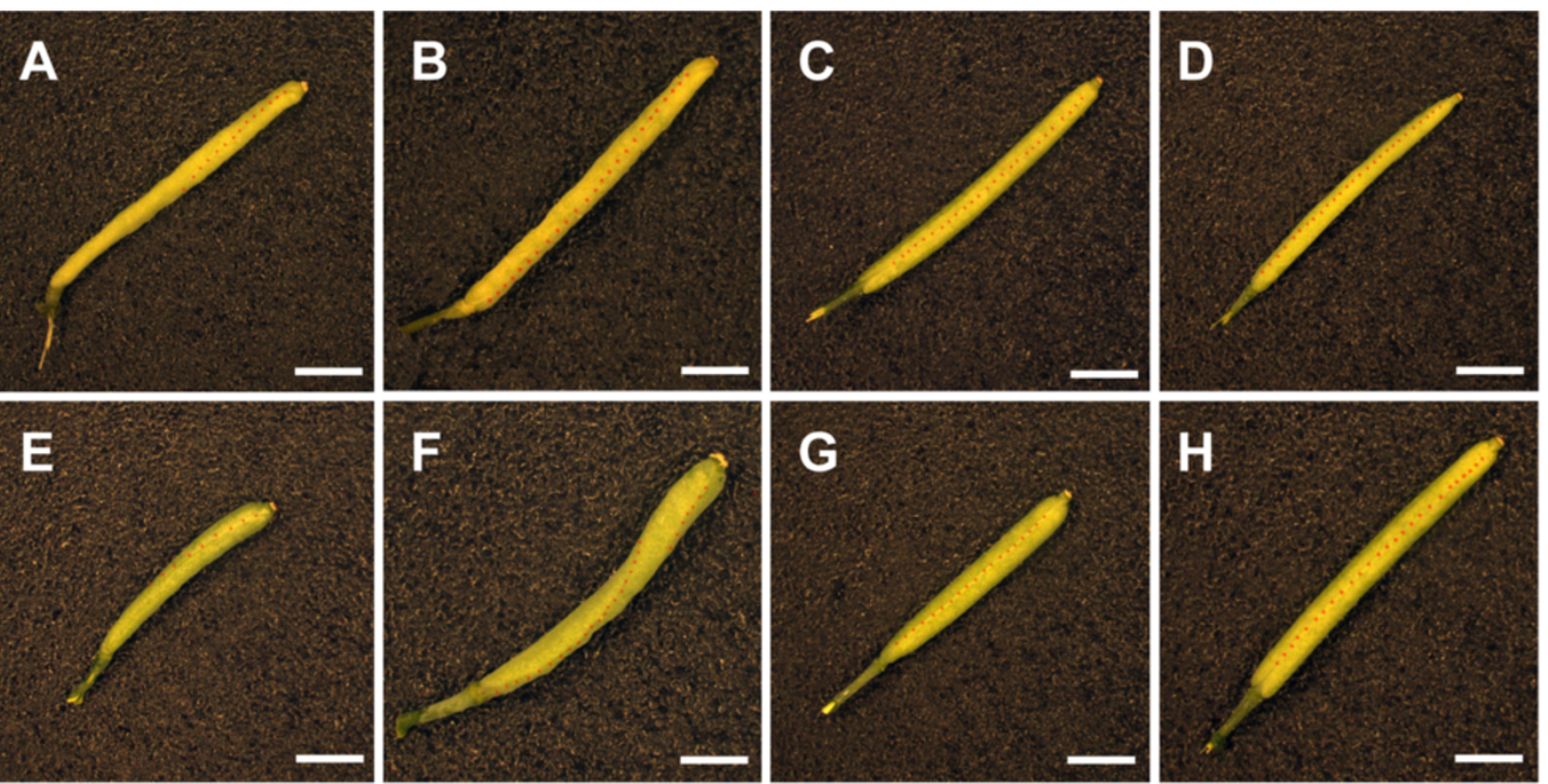

Figure 4 Silique morphology in WT, an mutants and rescued lines. Twisted siliques are evident in the an-1 mutant (A), an-2 mutant (B), doq-1 mutant (E) and the an-EM1 mutant (F). The an-2 355::YFP:AN \#2 line (C) and an-2 355::YFP:AN \#4 line (D) exhibit normal silique morphology, compared to WT Ler $(\mathbf{G})$ and WT Col-0 $(\mathbf{H})$. The replums are highlighted by dashed lines to indicate where twisting is present. Scale bars: $2 \mathrm{~mm}$. 

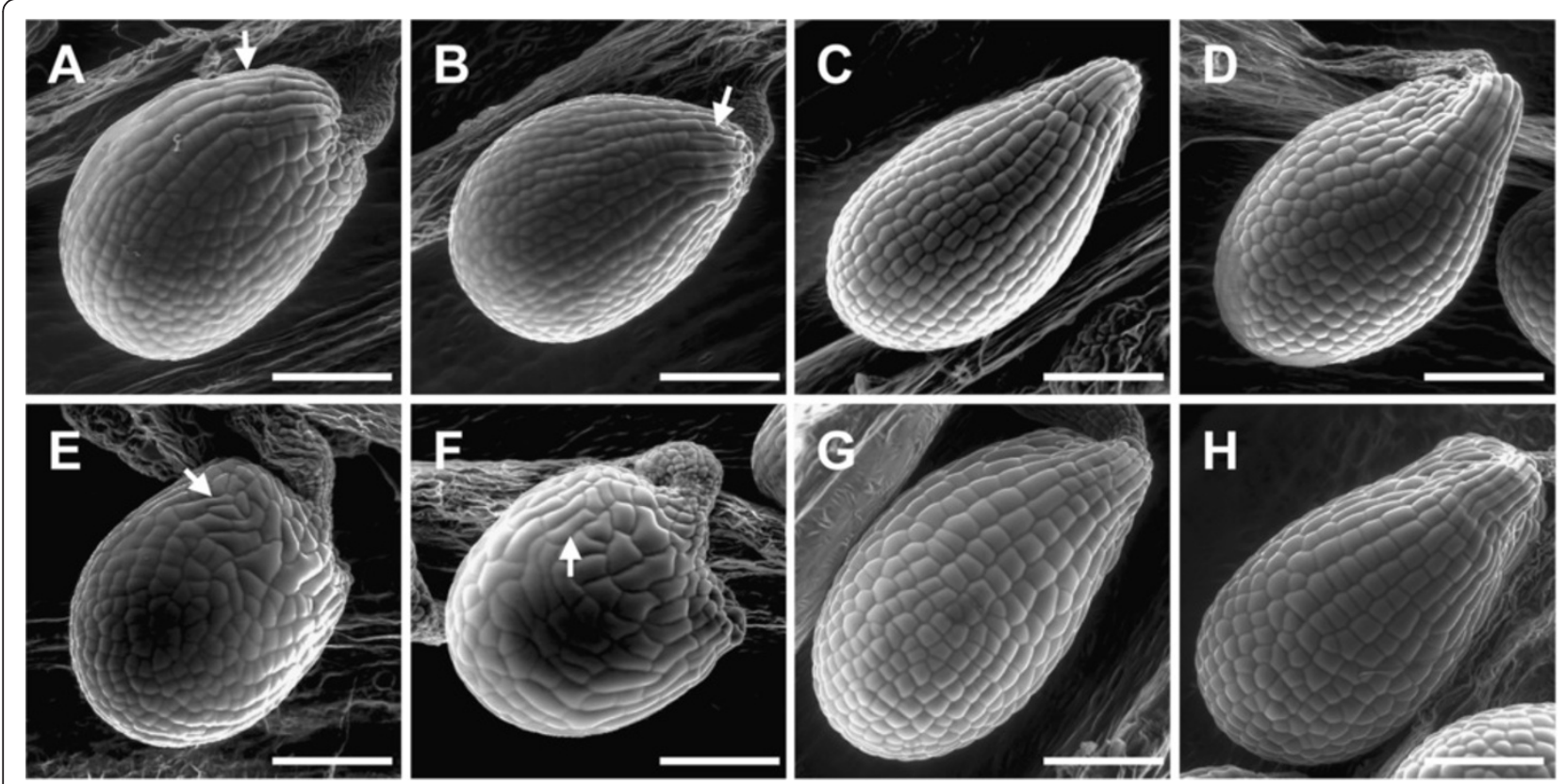

Figure 5 Ovule shape in WT, an mutants and rescued lines. Ovules in the an-1 mutant (A), the an-2 mutant (B), the an-2 355 ::YFP:AN \#2 line (C), the an-2 35S::YFP:AN \#4 line (D), the doq-1 mutant $(\mathbf{E})$, the an-EM1 mutant $(\mathbf{F})$, WT Ler $(\mathbf{G})$ and WT Col-0 $(\mathbf{H})$. Arrows point to regions with aberrant cell division. Scale bars: $100 \mu \mathrm{m}$.

$A N$ is involved in a broad variety of cellular processes $A N$ was initially identified by its narrow leaf phenotype and trichome phenotype [14]. The closer analysis of the narrow leaf phenotypes suggested a role in cell polarity of leaf cells, such that the length of individual cells was increased and the width reduced $[15,20]$. As cell number is also changed an additional role in cell division control was postulated. The characterization of the trichome phenotype placed $A N$ in a regulatory network controlling branching initiation $[12,13]$. In this context, a conspicuous lack of microtubule accumulation in the branch initiation zone of the developing trichome cell suggested a role of
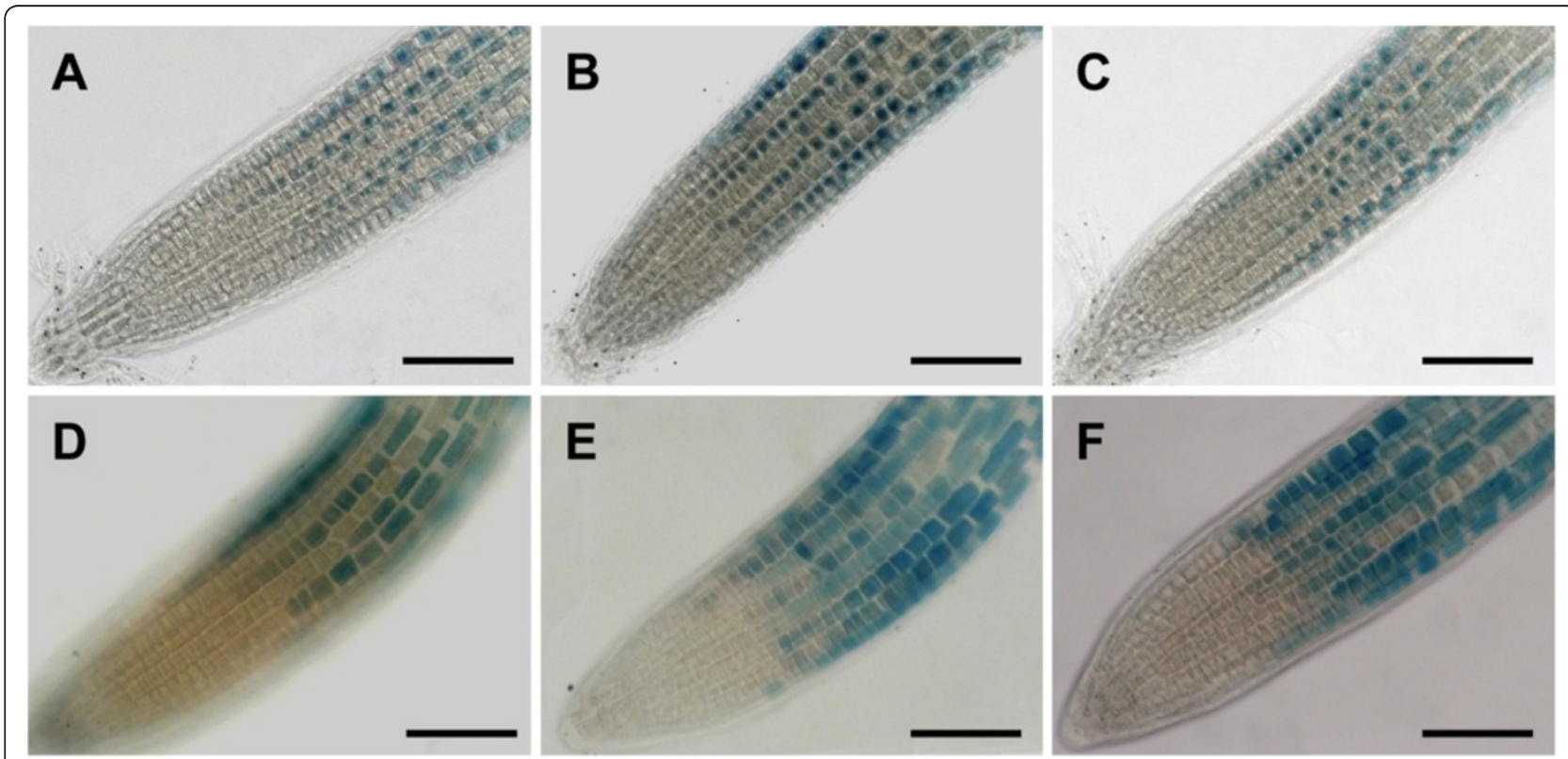

Figure 6 GL2:GUS expression pattern in WT and an mutants. The expression pattern of the GL2 promoter GUS fusion is shown in Ler (A), the doq-1 mutant (B), the an-EM1 mutant (C), Col-0 (D), the an-1 mutant (E), the an-2 mutant (F), Scale bars: $100 \mu m$. 


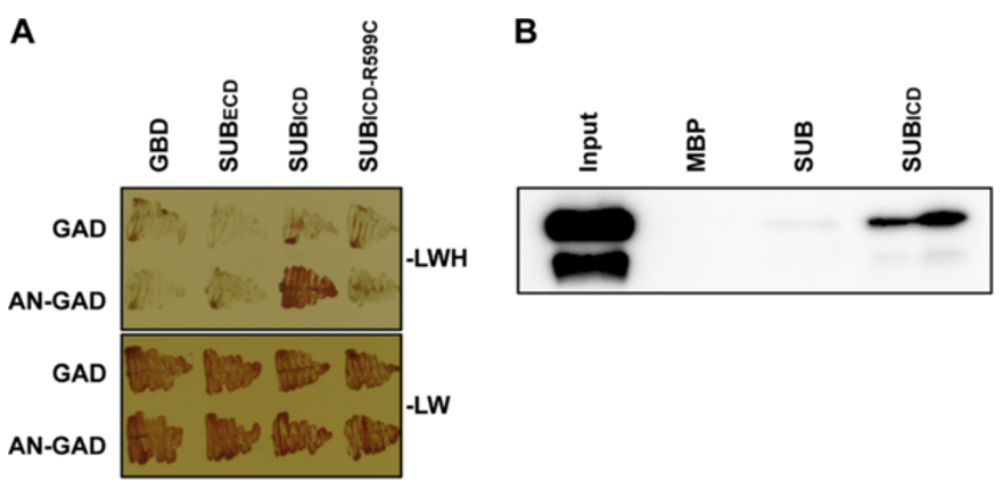

Figure 7 SUB can physically interact with AN. (A) Results of a yeast two-hybrid assay are depicted. The top panel shows that only cells carrying both plasmids encoding SUBICD and AN can grow on drop-out medium lacking leucine, tryptophan, and histidine (-LWH). Interaction depends on the presence of a functional SUBICD as indicated by the absence of signal in the variant SUBICD-R599C which mimics a strong sub mutation [6]. As a control all transformants can grow on medium lacking leucine and tryptophan (-LW) (bottom panel). Assays were done in the presence of 2.5 mM 3-amino-1,2,4-triazole (3-AT). GAD and GBD denote empty vector controls. (B) Western blot analysis of in vitro pull-down experiments to test MBP:SUB (full length) or MBP:SUBICD binding to GST:AN. AN was detected using a specific anti-AN antibody. The antibody detects two bands, both of which represent intact AN, as shown by MALDI-TOF analysis, suggesting two different conformations. GST:AN does not interact with MBP alone.

AN in the control of microtubule organization [21]. The new $s l m$ phenotypes of an mutants reported in this study indicate that $A N$ is involved in a SUB dependent signal transduction cascade. This function is separate from the first two functional aspects derived from leaf shape and trichome phenotypes, as sub mutants do not share these phenotypic aspects [2]. Such a broad spectrum of functions is compatible with the proposed biochemical functions of the AN protein. The $A N$ gene encodes a protein with homology to CtBP/BARS $[21,22]$. CtBPs (C-terminal Binding Protein) were initially discovered as proteins binding to the $\mathrm{C}$-terminal domain of adenovirus EA-1 [23]. CtBPs have been described as co-repressors for many transcriptional repressors carrying a PxDLS or RRT protein motif $[24,25]$. A possible function of $\mathrm{AN}$ as a corepressor is the finding that micro-array experiments revealed many genes that are transcriptionally regulated by $\mathrm{AN}[2,22]$. CtBP/BARS were independently identified as Brefeldin A ADP ribosylated substrates (BARS) [17]. A possible Golgi-related function is supported by the finding that CtBP can induce constriction in Golgi tubules [26] and membrane fission [27]. In support of such a function AN was reported to act outside the nucleus [28].

\section{Role of $A N$ in SUB-mediated signal transduction}

How does $A N$ fit into the $S U B$-mediated signaling pathway? $A N$ is unlikely to be a direct or indirect transcriptional target of SUB signaling. For example, SUB expression is only minimally altered in $d o q-1$ flowers at various stages [2]. In addition, a 35S::SUB transgene failed to rescue the phenotype of doq-1 mutants indicating that $S U B$ is not directly regulated at the transcriptional level by $A N$. At the same time, $A N$ expression was not found to differ between floral tissue of wild type, sub-1 and other slm mutants [2] (data not shown). These observations render it unlikely that $A N$ and $S U B$ regulate each other's activity at the transcriptional level.

Subcellular localization of a functional SUB:EGFP fusion protein was found to be restricted to the plasma membrane [6,7]. A functional AN:GFP fusion protein was recently reported to reside in the cytoplasm and in punctate compartments around the trans-Golgi network (TGN) [28]. The TGN localization of AN led the authors to suggest a Golgi-related role for this protein, possibly in membrane trafficking. These results are compatible with at least two possibilities of how AN may fit into the SUB signaling mechanism. In the first scenario AN could mediate membrane trafficking of SUB, a view that is also indirectly supported by the finding that $Q K Y$ encodes a putative membrane-localized protein thought to function in membrane-associated $\mathrm{Ca}^{2+}$ - and phospholipiddependent signaling [2]. However, this model does not fit the data presented in this study as signal distribution of a functional SUB::SUB:EGFP reporter was found to be unaltered in $d_{0}-1$ roots. In an alternative scenario, the cytoplasmic distribution of AN would allow its direct interaction with the intracellular domain of SUB.

We currently favor this notion as results from the yeast two-hybrid and in vitro pull-down assays suggest direct interaction between SUB and AN proteins at the plasma membrane. This interaction could then be necessary to control further downstream events of SUB signal transduction. AN is likely to mediate only some aspects of SUB signaling as there is a difference in the strength between for example the ovule phenotypes of sub and doq-1 mutants and stem twisting is nearly 

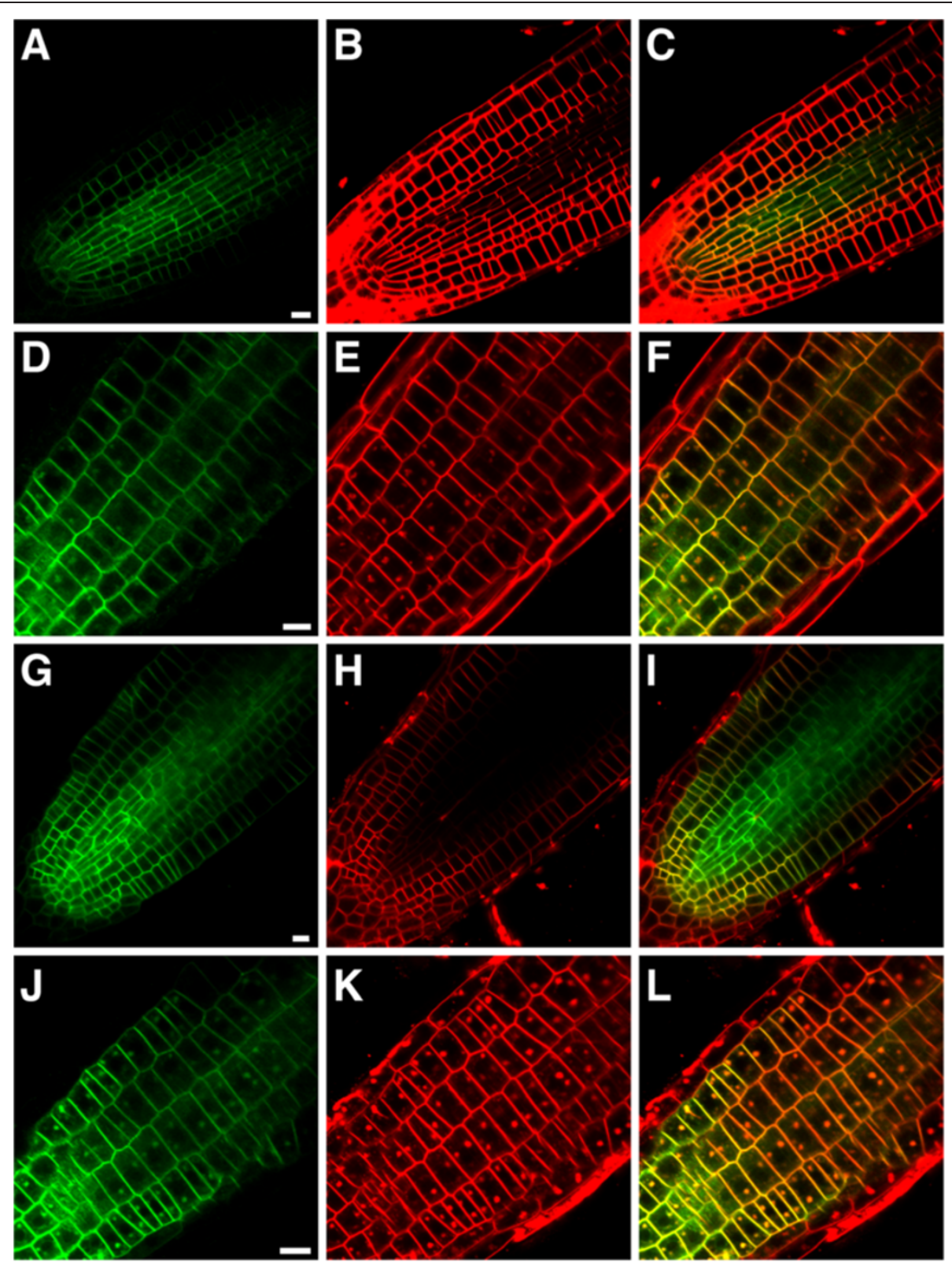

Figure 8 SUB::SUB:EGFP expression in five-days-old root tips of WT and doq-1 mutants in the absence and presence of BFA. Confocal micrographs are shown. Two independent lines transgenic for a functional SUB:SUB:EGFP reporter [6] exhibiting a GFP-based signal were analyzed for each genotype. GFP-based signal is shown in (A, D, G, J), FM4-64-based counterstain in (B, E, H, K) and the overlay in (C, F, I, L). Mid-optical section through a wild-type root tip highlights the plasma membrane localization of SUB:EGFP (A-C). Tangential optical section through the epidermis of a wild-type root tip treated with $50 \mu \mathrm{m}$ BFA for 30 minutes reveals the typical dotted structures that indicate the presence of BFA compartments (D-F). A similar set is shown for untreated doq-1 roots (G-I) and BFA-treated doq-1 roots (J-L). Scale bars: $10 \mu \mathrm{m}$.

absent in $d o q-1$ or $a n-2$ (Figure 9). Moreover, there is only partial overlap in misexpressed genes between $s u b$ and doq-1 flowers [2]. With respect to plant height AN appears to be part of the SUB mechanism that interacts with the LRR-RLK ER. It was proposed that ER and $S U B$ signaling converge either at the level of the receptors or at some level more downstream in the mechanism [6]. Thus, it will be interesting to resolve exactly how SUB,
AN and ER relate to each other in future studies on SUB signaling.

\section{Conclusions}

In this study we showed that phenotypes of the $s / m$ mutant doq-1 and the trichome and leaf shape mutant an overlap. In addition, we showed that doq-1 is allelic to an. We further demonstrated that $d o q-1$ is not an 


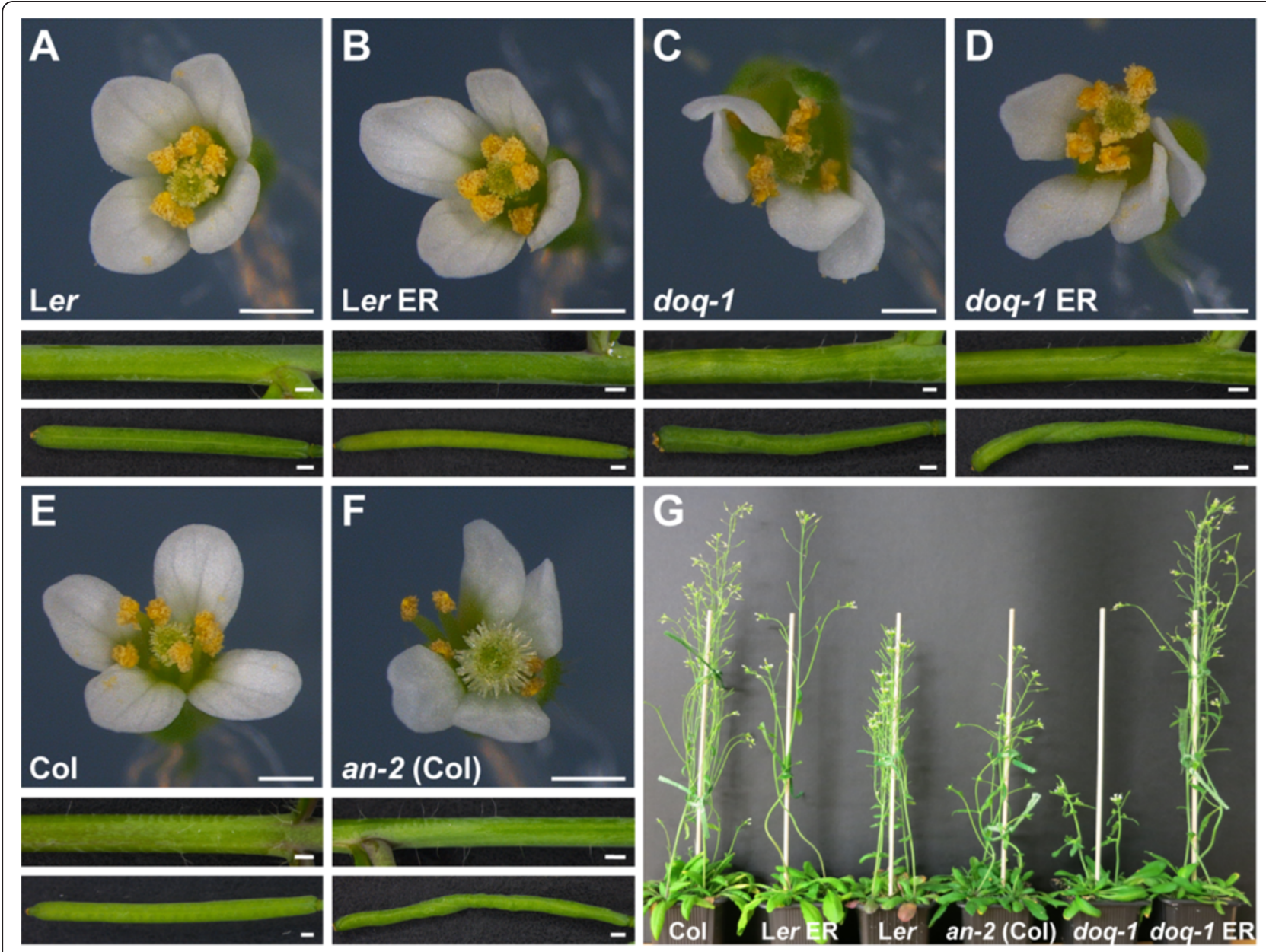

Figure 9 The doq-1 above-ground phenotype in the presence of functional ERECTA. Comparison of wild type, an-2 (Col), doq-1 (Ler) and doq-1 plants transgenic for Col ER-containing plasmid pKUT196. (A-F) Morphology of flowers (upper panel), stems (central panel) and siliques (bottom panel). (A) Wild-type Ler. (B) Transgenic Ler pKUT196. (C) doq-1 mutant. Note the aberrant flower and silique morphology. Stem twisting is very mild if present at all. (D) Transgenic doq-1 pKUT196. Note the irregular flower and silique morphology. Stem morphology is essentially normal (compare with F). (E) Col wild type. (F) Col an-2 mutant. Note the aberrant flower and silique morphology. Stem twisting is nearly normal. (G) Plant height comparisons of six-week-old pKUT196 transgenic plants in comparison to wild type and mutant reference lines. Note the height reduction in an-2 (Col) plants. Scale bars: $0.5 \mathrm{~mm}$.

atypical an allele but that all tested an mutants show previously undescribed $s l m$ aspects. The data reveal a broader range of biological functions for $A N$ than previously appreciated. Finally, our data reveal the possibility that SUB and AN interact directly. Taken together, the presented evidence suggests a role for AN in tissue morphogenesis mediated by the atypical receptor-like kinase SUB.

\section{Methods}

\section{Plant work and genetics}

The following Arabidopsis thaliana (L.) Heynh. lines were used in this study: Columbia (Col-0) and Landsberg (erecta mutant) (Ler) wild-type strains, doq-1 [2], an-1, an-2 [22], an-EM1 [21], an-2 35S::YFP:AN. an-2 35S::YFP:AN plants were generated by cloning the CDS of the YFP:AN fusion into the PPAMPAT vector containing the $35 \mathrm{~S}$ promoter (GenBank accession AY027531). Plasmid pKUT196 was described previously [19]. Plant transformation was performed according to the floral dip method [29].

Plants were grown on soil at $24^{\circ} \mathrm{C}$ with 16 hours of light per day. The GL2:GUS line (Ler) [16] was introduced into an mutants by backcrosses. For GUS assays, plants were grown on MS plates for 4 days.

Using a Ler/Col mapping population doq-1 was localized to the upper end of chromosome 1 between markers F10O3(481D) and NF21M12 [2]. Further mapping revealed an interval of $330.6 \mathrm{~kb}$. The final Northern marker 96_(BccI) was located at chromosomal position 96771 (one recombinant left). 96_(BccI) is a CAPS 
marker which yields the following products after PCR with primers 96_(BccI)_F (GGGCTTTGATTTGATTG TGG) and 96_(BccI)_R (AAGAGAGGAGTGCAGCC AAA) and BccI digestion: Ler - 498 bp, Col - 254, 244 bp. The final Southern marker was NT7123 (chromosomal position: 427,343 bp, 3 recombinants left). NT7123 is a SSLP marker that, following PCR with primers NT7123_F (GTGTCCTTTTTTCTCAAC GATG) and NT7123_R (CATGCACGTACGATTTGT TTAAC), yielded the following products on a $3.5 \%$ agarose gel: Ler , <199 bp; Col, 199 bp.

\section{Yeast two-hybrid assay}

The Matchmaker yeast two-hybrid system (Clontech) was employed and experimental procedures followed the manufacturer's recommendations. The pACT-AN construct was described previously [21]. For the generation of pGBKT7-SUBICD, the intracellular part of SUB coding sequence [1] was amplified from cDNA using primers SUBintra_F (CATGCCATGGATAACCGATAT TACAGTG) and SUBintra_R (ATCGGTCGACAATA AACTATTGCTTCTG). The PCR product was digested with NcoI/SalI and cloned into NcoI/SalI digested pGBKT7. The R599C mutation was introduced into pGBKT7-SUBICD by the QuikChange II XL site-directed mutagenesis kit according to the manufacturer's recommendations (Agilent Technologies) by using primers 35SsubR599Cf (AAGAAGCTCACTTGGAATGTATGTA TAAATATTGCATTAGGAGCTTC) and 35SsubR599Cr (G A AG C T CCTAATGCAATATTTATACATACATTC CAAGTGAGCTTCTT). For the cloning of pGBKT7SUBECD, the extracellular part of SUB coding sequence was amplified from cDNA using primers SUB Extra/ Nde1_F (GCTCATATGACTAATCTACGAGATGTTTC GGCGA and SUB Extra/Xma1_R (TACCCGGGGTTGA GTGGACCAGAATTTTCCTGATC). The PCR product was digested with NdeI/XmaI and cloned into NdeI/XmaI digested pGBKT7. All PCR-based constructs were sequenced.

To assay possible interactions in yeast pGBKT7 plasmids containing SUBECD, SUBICD and SUBICDR599C were cotransformed with pACT or pACT-AN into yeast strain AH109. Transformants were selected after 3 days on SC medium lacking Leu and Trp (-LW) at $30^{\circ} \mathrm{C}$. To examine yeast two-hybrid interactions, the transformants were grown on solid SC medium lacking Leu and Trp (SC-LW) or Leu, Trp, and His (SC-LWH) at $30^{\circ} \mathrm{C}$.

\section{Generation of constructs for recombinant protein production}

$A N$ and SUB cDNA were cloned into gateway entry vector pDONR 201. The following primers were used for gateway cloning: for amplifying $A N$ cDNA: GGGGACA
AGTTTGTACAAAAAAGCAGGCTTCATGAGCAAGA TCCGTTCG and GGGGACCACTTTGTACAAGAAAG CTGGGTCT TAATCGATCCAACGTGTGATAC; for amplification of the full length $S U B$ cDNA: GGGGA CAAGTTT GTACAAAAA AGCAGGCTTCATGAGCT TTACAAGATGGGAAGTGT and GGGGACCACTTT GTACAAGAAAGCTGGGTCTTAGATCATATGTTGA AGATCTTGG; for the amplification of the ICD version of SUB: GGGGACAAGTTTGTACAAAAAAGCAGGCTT CatgTATAACCGATATTACAGTGGAGC and GGGGA CCACT T TGTACAAGAAAGCTGGGTCTTAGATCAT ATGTTGAAGATCTTGG. AN cDNA was cloned into pGEX2TMGW (GE Healthcare) to generate an N-terminal fusion with Glutathion-S-Transferase (GST:AN) and the SUB and SUBICD cDNAs were cloned into pETG-40a (EMBL, Heidelberg) to generate an $\mathrm{N}$-terminal fusion with maltose-binding protein (MBP:SUB, MBP:SUBICD).

\section{Antibody generation}

To produce anti-AN antibody, AN was expressed as a GST-fusion protein in E.coli. The protein was purified and used to generate antibodies in rabbit (Pineda AntikörperService; Berlin, Germany). The antibody serum was affinity purified and checked for its specificity by MALDI-TOF analysis.

\section{In vitro pull-down assay}

Interactions between SUB and AN were studied using purified proteins that were expressed in bacteria. The bacterial cells BL21-CodonPlus (DE3)-RIL containing the IPTG inducible constructs (MBP-Full length SUB, MBP-SUBICD and GST-AN) were grown at $37^{\circ} \mathrm{C}$ and $220 \mathrm{rpm}$ to an OD 600 of 0.8-0.9 and then the cultures were induced by adding IPTG to final concentration of $1 \mathrm{mM}$. The induced cells were then grown further for 5 hours (at $37^{\circ} \mathrm{C}$ for GST-tagged $\mathrm{AN}$ constructs and $20^{\circ} \mathrm{C}$ for MBP-tagged constructs) and cells were harvested by centrifugation. Cells were lysed in Tris-lysis buffer (Tris (pH 7.5) $50 \mathrm{mM}, \mathrm{NaCl}(100 \mathrm{mM})$, EDTA $(1 \mathrm{mM})$, EGTA (1 $\mathrm{mM})$, NP-40 (1\%), Lysozyme $(200 \mu \mathrm{g} / \mathrm{ml})$, DTT $(1 \mathrm{mM})$, Protease inhibitor cocktail (Sigma)) and sonicated three times for one minute each. The supernatant was collected by centrifugation at $4^{\circ} \mathrm{C}$. MBP-tagged proteins were purified by incubation with amylose resin overnight at $4^{\circ} \mathrm{C}$. After several washings, part of the resin was boiled with SDS-PAGE gel loading buffer to get purified protein for analysis. The remaining resin was used for incubation with equal amounts of GST:AN lysate for 4 hours at $4^{\circ} \mathrm{C}$ followed by several washings. Finally, the beads were boiled in SDS-PAGE gel loading buffer at $96^{\circ} \mathrm{C}$ for $10 \mathrm{~min}$ and equal amounts were loaded on a gel followed by western blotting. Detection was done using primary anti-AN antibody and secondary anti-rabbit antibody using SuperSignal 
West Femto Maximum Sensitivity Substrate (Thermo Scientific).

\section{Histochemical analysis, microscopy and BFA treatments} Histochemical localization of $ß$-glucuronidase (GUS) activity in whole-mount tissues was performed as described previously [16]. Scanning Electron Microscopy was made using a Quanta 250 FEG (FEI) microscope under low vacuum conditions without any fixation steps. Confocal laser scanning microscopy and BFA treatments were performed as reported previously [6].

\section{Competing interests}

The authors declare that they have no competing interests.

\section{Authors' contributions}

$L F, M H, K S$ designed the study. YB, LF, PV and HB performed experiments. $\mathrm{YB}, \mathrm{LF}, \mathrm{PV}, \mathrm{HB}, \mathrm{MH}$ and $\mathrm{KS}$ analyzed the data. $\mathrm{MH}$ and $\mathrm{KS}$ wrote the manuscript. All authors read and approved the final manuscript.

\section{Acknowledgements}

We thank B. Ülker (MPIZ, Cologne) for providing the pAMPAT-GW vector and K. Torii for the pKUT196 plasmid. We also thank members of the Hülskamp and Schneitz labs for stimulating discussions. This work was funded through grants SCHN 723/6-1 and SFB924 (TP A2) from the German Research Council (DFG) to KS. YB was funded by the International Max Planck Research School "Molecular Basis of Plant Development and Environmental Interaction". HB was funded by an IGSDHD fellowship.

\section{Author details}

'Botanisches Institut III, Universität Köln, Zülpicher Straße 47b, Köln 50674, Germany. ${ }^{2}$ Entwicklungsbiologie der Pflanzen, Wissenschaftszentrum Weihenstephan, Technische Universität München, Emil-Ramann-Str. 4, Freising 85354, Germany. ${ }^{3}$ Present address: Department of Plant Microbe Interactions, Max Planck Institute for Plant Breeding Research, Carl-von-Linné-Weg 10, Köln 50829, Germany. ${ }^{4}$ Present address: School of Biological Sciences, Monash University, Melbourne, VIC 3800, Australia.

Received: 24 January 2013 Accepted: 29 January 2013

Published: 31 January 2013

\section{References}

1. Chevalier D, Batoux M, Fulton L, Pfister K, Yadav RK, Schellenberg M, Schneitz K: STRUBBELIG defines a receptor kinase-mediated signaling pathway regulating organ development in Arabidopsis. Proc Natl Acad Sci USA 2005, 102:9074-9079.

2. Fulton L, Batoux M, Vaddepalli P, Yadav RK, Busch W, Andersen SU, Jeong $S$, Lohmann JU, Schneitz K: DETORQUEO, QUIRKY, and ZERZAUST represent novel components involved in organ development mediated by the receptor-like kinase STRUBBELIG in Arabidopsis thaliana. PLoS Genet 2009, 5:e1000355.

3. Kwak SH, Shen R, Schiefelbein J: Positional signaling mediated by a receptor-like kinase in Arabidopsis. Science 2005, 307:1111-1113.

4. Kwak SH, Schiefelbein J: The role of the SCRAMBLED receptor-like kinase in patterning the Arabidopsis root epidermis. Dev Biol 2007, 302:118-131.

5. Yadav RK, Fulton L, Batoux M, Schneitz K: The Arabidopsis receptor-like kinase STRUBBELIG mediates inter-cell-layer signaling during floral development. Dev Biol 2008, 323:261-270.

6. Vaddepalli P, Fulton L, Batoux M, Yadav RK, Schneitz K: Structure-function analysis of STRUBBELIG, an Arabidopsis atypical receptor-like kinase involved in tissue morphogenesis. PLoS One 2011, 6:e19730.

7. Kwak SH, Schiefelbein J: A feedback mechanism controlling SCRAMBLED receptor accumulation and cell-type pattern in Arabidopsis. Curr Biol 2008, 18:1949-1954.
8. Shiu S-H, Bleecker AB: Receptor-like kinases from Arabidopsis form a monophyletic gene family related to animal receptor kinases. Proc Natl Acad Sci USA 2001, 98:10763-10768.

9. Eyüboglu B, Pfister K, Haberer G, Chevalier D, Fuchs A, Mayer KF, Schneitz K: Molecular characterisation of the STRUBBELIG-RECEPTOR FAMILY of genes encoding putative leucine-rich repeat receptor-like kinases in Arabidopsis thaliana. BMC Plant Biol 2007, 7:16.

10. Castells E, Casacuberta JM: Signalling through kinase-defective domains: the prevalence of atypical receptor-like kinases in plants. J Exp Bot 2007, 58:3503-3511.

11. Gish LA, Clark SE: The RLK/Pelle family of kinases. Plant J 2011, 66:117-127.

12. Folkers U, Berger J, Hülskamp M: Cell morphogenesis of trichomes in Arabidopsis: differential control of primary and secondary branching by branch initiation regulators and cell growth. Development 1997, 124:3779-3786.

13. Hülskamp M, Misera S, Jürgens G: Genetic dissection of trichome cell development in Arabidopsis. Cell 1994, 76:555-566.

14. Redei GP: Single locus heterosis. Z Vererbungs/ 1962, 93:164-170.

15. Tsuge $T$, Tsukaya H, Uchimiya H: Two independent and polarized processes of cell elongation regulate leaf blade expansion in Arabidopsis thaliana (L.) Heynh. Development 1996, 122:1589-1600

16. Masucci JD, Rerie WG, Foreman DR, Zhang M, Galway ME, Marks MD, Schiefelbein JW: The homeobox gene GLABRA2 is required for positiondependent cell differentiation in the root epidermis of Arabidopsis thaliana. Development 1996, 122:1253-1260.

17. Spanò S, Silletta MG, Colanzi A, Alberti S, Fiucci G, Valente C, Fusella A, Salmona M, Mironov A, Luini A, Corda D: Molecular cloning and functional characterization of brefeldin A-ADP-ribosylated substrate. A novel protein involved in the maintenance of the Golgi structure. J Biol Chem 1999, 274:17705-17710.

18. Torii KU, Mitsukawa N, Oosumi T, Matsuura Y, Yokoyama R, Whittier RF, Komeda Y: The Arabidopsis ERECTA gene encodes a putative receptor protein kinase with extracellular leucine-rich repeats. Plant Cell 1996, 8:735-746.

19. Godiard L, Sauviac L, Torii KU, Grenon O, Mangin B, Grimsley NH, Marco Y: ERECTA, an LRR receptor-like kinase protein controlling development pleiotropically affects resistance to bacterial wilt. Plant J 2003, 36:353-365.

20. Tsukaya H, Tsuge T, Uchimiya H: The cotyledon: a superior system for studies of leaf development. Planta 1994, 195:309-312.

21. Folkers U, Kirik V, Schobinger U, Falk S, Krishnakumar S, Pollock MA, Oppenheimer DG, Day I, Reddy AS, Jürgens G, Hülskamp M: The cell morphogenesis gene ANGUSTIFOLIA encodes a CtBP/BARS-like protein and is involved in the control of the microtubule cytoskeleton. EMBO $J$ 2002, 21:1280-1288.

22. Kim GT, Shoda K, Tsuge T, Cho KH, Uchimiya H, Yokoyama R, Nishitani $K$, Tsukaya H: The ANGUSTIFOLIA gene of Arabidopsis, a plant CtBP gene, regulates leaf-cell expansion, the arrangement of cortical microtubules in leaf cells and expression of a gene involved in cell-wall formation. EMBO J 2002, 21:1267-1279.

23. Boyd JM, Subramanian T, Schaeper U, La Regina M, Bayley S, Chinnadurai G: A region in the $\mathrm{C}$-terminus of adenovirus $2 / 5 \mathrm{E} 1$ a protein is required for association with a cellular phosphoprotein and important for the negative modulation of T24-ras mediated transformation, tumorigenesis and metastasis. EMBO J 1993, 12:469-478.

24. Chinnadurai $\mathrm{G}$ : CtBP, an unconventional transcriptional corepressor in development and oncogenesis. Mol Cell 2002, 9:213-224.

25. Quinlan KG, Nardini M, Verger A, Francescato P, Yaswen P, Corda D, Bolognesi M, Crossley M: Specific recognition of ZNF217 and other zinc finger proteins at a surface groove of C-terminal binding proteins. Mol Cell Biol 2006, 26:8159-8172.

26. Weigert R, Silletta MG, Spano S, Turacchio G, Cericola C, Colanzi A, Senatore S, Mancini R, Polishchuk EV, Salmona M, Facchiano F, Burger KN, Mironov A, Luini A, Corda D: CtBP/BARS induces fission of Golgi membranes by acylating lysophosphatidic acid. Nature 1999, 402:429-433.

27. Bonazzi M, Spano S, Turacchio G, Cericola C, Valente C, Colanzi A, Kweon HS, Hsu WW, Polishchuck EV, Polishchuck RS, Sallese M, Pulvirenti T, Corda D, Luini A: CtBP3/BARS drives membrane fission in dynamin-independent transport pathways. Nat Cell Biol 2005, 7:570-580. 
28. Minamisawa N, Sato M, Cho KH, Ueno H, Takechi K, Kajikawa M, Yamato KT, Ohyama K, Toyooka K, Kim GT, Horiguchi G, Takano H, Ueda T, Tsukaya H: ANGUSTIFOLIA, a plant homolog of CtBP/BARS, functions outside the nucleus. Plant J 2011, 68:788-799.

29. Clough SJ, Bent AF: Floral dip: a simplified method for Agrobacteriummediated transformation of Arabidopsis thaliana. Plant J 1998, 16:735-743.

doi:10.1186/1471-2229-13-16

Cite this article as: Bai et al: ANGUSTIFOLIA is a central component of tissue morphogenesis mediated by the atypical receptor-like kinase STRUBBELIG. BMC Plant Biology 2013 13:16.

\section{Submit your next manuscript to BioMed Central and take full advantage of:}

- Convenient online submission

- Thorough peer review

- No space constraints or color figure charges

- Immediate publication on acceptance

- Inclusion in PubMed, CAS, Scopus and Google Scholar

- Research which is freely available for redistribution 